\title{
Obesidade e covid-19: duas epidemias que, combinadas, repercutem em mau prognóstico de pacientes
}

\author{
Obesity and covid-19: two epidemics that, combined, result in poor patient \\ prognosis
}

DOI: $10.46919 / \operatorname{archv2n3-036}$

Recebimento dos originais: 01/01/2021

Aceitação para publicação: 31/03/2021

Iluskhanney Gomes de Medeiros Nóbrega Miranda

UNINASSAU - Centro Universitário Maurício de Nassau, Nutrição

E-mail: yluska.gmn@gmail.com

\author{
Dr. Juscelino Kubitschek Bevenuto da Silva \\ UNINASSAU-Professor do Centro Universitário Maurício de Nassau/Campina Grande-PB \\ E-mail: nutribevenuto@ hotmail.com \\ Ingrid Rafaella Mauricio Silva Reis \\ Nutricionista, UPE - Universidade de Pernambuco, Mestranda em biociências, UNIVASF \\ E-mail: ingridrafa.15@gmail.com

\section{Isadora Garcia Pires} \\ Nutricionista - UNIPAM - Centro Universitário de Patos de Minas \\ E-mail: isadoragarciapires@gmail.com

\section{Dr. José Guedes da Silva Júnior} \\ UNINASSAU-Professor do Centro Universitário Maurício de Nassau/Campina Grande-PB \\ E-mail: zeguedescrizant@gmail.com
}

\author{
M.Sc. Áquila Matheus de Souza Oliveira \\ Doutorando em Alimentos e Nutrição-Unicamp \\ E-mail: Aquila.ufc@gmail.com
}

\section{RESUMO}

A obesidade, em si, trata-se de uma doença crônica, inflamatória, de origem multifatorial onde diversos fatores podem estar envolvidos, como: hereditariedade, hábitos adquiridos ao longo da vida e fatores ambientais, sendo considerada uma condição pandêmica no mundo. Indivíduos que apresentam o dignóstico nutricional de obesidade e, também, outros fatores de riscos que pioram o quadro, como já mencionado, possuem maiores chances de desenvolverem outras comorbidades, tais como, cardiopatias, diabetes, câncer, hipertensão e problemas respiratórios, patologias essas vulneráveis a levarem a um prognóstico desfavorável quando associado com a infecção pelo COVID 19. Este artigo de revisão bibliográfica teve como objetivo verificar de que forma a obesidade pode ser um fator de risco para um mau prognóstico diante de pacientes acometidos pelo novo coronavírus. A seleção e localização das referências que fundamentassem esta revisão foram retiradas das bases de dados PubMed/LILACS e da biblioteca eletrônica SciELO, utilizando também a Biblioteca Virtual em Saúde (BVS), a fim de identificar artigos científicos publicados no período de 2020 e 2021, utilizando como descritores: obesidade e covid19; fatores de risco e Covid-19; obesidade e Sars-Cov-2 e suas versões em inglês. A literatura evidencia 
que o estado obesogênico pode conduzir para um mau prognóstico em pacientes obesos e infectados com SARS-CoV-2. Podemos observar que o grau de comprometimento da saúde é independente do grau de obesidade, sendo que a medida que este aumenta, os riscos de internação e intubação ou morte em comparação com os pacientes sem obesidade, aumentam substancialmente. Algumas condições exercem grande influência sobre o mal prognóstico, são exemplos, a distribuição do tecidoa adiposo, quando essa reserva se acumula na parte visceral, contribui para resistência a insulina, dislipidemia, aterosclerose, inflamação, diabetes II, síndrome metabólica e maior risco de DCV e tatmbém, o próprio estado inflamatório característico da obesidade.

Palavras-chave: Fator de Risco, SARS-CoV-2, Estado nutricional, Obesidade.

\begin{abstract}
Obesity, in itself, is a chronic, inflammatory disease, of multifactorial origin where several factors may be involved, such as: heredity, habits acquired throughout life and environmental factors, being considered a pandemic condition in the world. Individuals who have the nutritional status of obesity and, also, other risk factors that worsen the condition, as already mentioned, are more likely to develop other comorbidities, such as heart disease, diabetes, cancer, hypertension and respiratory problems, pathologies that are vulnerable to lead to an unfavorable prognosis when associated with the infection by COVID 19. This Bibliographic Review article aimed to verify how obesity can be a risk factor for a poor prognosis for patients affected by the new coronavirus. The selection and location of the references that supported this review were taken from the PubMed / LILACS databases and the SciELO electronic library, also using the Virtual Health Library (VHL), in order to identify scientific articles published in the period 2020 and 2021, using as descriptors: obesity and covid-19; risk factors and Covid-19; obesity and Sars-Cov-2 and their English versions. The literature shows that the obesogenic status can lead to a poor prognosis in obese patients and those infected with SARS-CoV-2. We can observe that the degree of health impairment is independent of the degree of obesity, and as it increases, the risks of hospitalization and intubation or death compared to patients without obesity, increase substantially. Some conditions have a great influence on the poor prognosis, for example, the distribution of adipose tissue, when this reserve accumulates in the visceral part, contributes to insulin resistance, dyslipidemia, atherosclerosis, inflammation, diabetes II, metabolic syndrome and increased risk of CVD and also, the inflammatory state characteristic of obesity.
\end{abstract}

Keywords: Risk Factor, SARS-CoV-2, Nutritional status, Obesity.

\title{
1 INTRODUÇÃO
}

Grandes mudanças ocorreram no processo saúde/doença nos últimos anos, principalmente, a partir da metade do século XX, no qual foi percebido e se instalado, de maneira grandiosa e significativa, uma transição nutricional, caracterizada por uma superposição de carências nutricionais globais como o sobrepeso e a obesidade, condições essas associadas com múltiplas consequências adversas à saúde (MELO et al., 2020).

O sobrepeso e a obesidade podem ser definidos como um distúrbio nutricional e metabólico, o qual é caracterizado pelo aumento da quantidade de gordura corporal, além do padrão aceitável de normalidade, resultando, assim, no aumento do peso corpóreo. Tais condições se integram ao grupo das doenças crônicas 
não transmissíveis (DCNT) e representam fatores de riscos importantes de morbimortalidade em populações adultas (MELO et al., 2020; DIAS et al., 2017).

A obesidade, em si, trata-se de uma doença crônica, inflamatória, de origem multifatorial onde diversos fatores podem estar envolvidos, como: hereditariedade, hábitos adquiridos ao longo da vida e fatores ambientais, sendo considerada uma condição pandêmica no mundo. No entanto, o consumo de calorias maior que o necessário é a principal característica para o quadro patológico (SBP, 2019).

A Organização Mundial de Saúde (OMS) define a obesidade com base no Índice de Massa Corporal (IMC) definido pelo cálculo do peso corporal, em quilogramas, dividido pela altura ao quadrado, em metros quadrados $\left(\mathrm{IMC}=\mathrm{kg} / \mathrm{h}^{2}(\mathrm{~m})\right)$, e também pelo risco de mortalidade associada. A obesidade é caracterizada quando o IMC encontra-se acima de $30 \mathrm{~kg} / \mathrm{m}^{2}$, salientando que ainda há uma estratificação dos graus de obesidade (graus I, II e III) (OMS, 1995).

Já em outro cenário, a doença do novo coronavírus "COVID-19”, nomeada pela Organização Mundial da Saúde (OMS) declarada como Pandemia Global em março de 2020, é considerada uma morbidade conduzida pelo vírus SARS-CoV-2 de RNA de cadeia positiva, o qual traz consigo consequências como efeitos deletérios ao sistema respiratório, e intestinal em alguns hospedeiros, acarretando desde sintomas leves até mortais (ABBAS et al., 2020; CUMMINGS et al., 2020).

Indivíduos que apresentam o dignóstico nutricional de obesidade e, também, outros fatores de riscos que pioram o quadro, como já mencionado, possuem maiores chances de desenvolverem outras comorbidades, tais como, cardiopatias, diabetes, câncer, hipertensão e problemas respiratórios, patologias essas vulneráveis a levarem a um prognóstico desfavorável quando associado com a infecção pelo COVID 19 (CARNEIRO \& DALL'IGNA, 2020).

Os efeitos de um IMC elevado na mortalidade de COVID-19 podem ser mais fortes para mulheres. Por outro lado, há evidências de prevalência 42 vezes maior para o desenvolvimento de pneumonia grave em pacientes obesos, comparando com aqueles em estado eutrófico, sendo o público masculino 4,4 vezes maior de progressão para a gravidade em relação ao feminino. De todo modo, chama-se a atenção para a obesidade manter-se associada a maiores complicações em diferentes estudos, em ambos os sexos (PETERS, SAE. et al., 2020; QINGXIAN, C. et al., 2020).

O chamado "Choque de pandemias", é um termo recentemente abordado, o qual traduz a associação da ocorrência da infecção pelo novo coronavírus e a obesidade, ou seja, refere-se a pacientes acometidos por duas doenças com perfil pandêmico simultaneamente (CARNEIRO \& DALL'IGNA, 2020).

Existem múltiplos mecanismos possíveis e potenciais que colocam a obesidade como um fator de risco agravante para infecção por SARS-CoV-2, dentre eles: a alta deposição de gordura corporal sobre as vísceras e órgãos, que pode está associado com a redução da função de proteção cardiorrespiratória, 
resultando em uma função pulmonar prejudicada; o estado inflamatório característico da obesidade, podendo esse ser o principal fator, entre outros (CARNEIRO \& DALL'IGNA, 2020).

Tendo em vista tais definições bem elucidadas diante da literatura, torna-se necessário realizar estudos diante da temática, uma vez que a pandemia da COVID-19 emergiu de forma ardilosa, trazendo consigo grande exponencial quando colidindo com outras pandemias globais mais antigas, a exemplo da obesidade, repercutindo em maior vulnerabilidade do quadro clínico dos portadores e mortalidade.

Diante disso, o objetivo desta revisão bibliográfica é verificar de que forma a obesidade pode ser um fator de risco para um mau prognóstico diante de pacientes acometidos pelo o novo coronavírus.

\section{METODOLOGIA}

Trata-se de uma pesquisa de revisão bibliográfica integrativa. Para assegurar a consecução do objetivo descrito, realizou-se um estudo exploratório e a seleção e localização das referências que fundamentassem esta revisão foram retiradas das bases de dados PubMed/LILACS e da biblioteca eletrônica SciELO, utilizando também a Biblioteca Virtual em Saúde (BVS), que integra as bases de dados acima citadas, a fim de identificar artigos científicos publicados no período de 2020 e 2021, utilizando os seguintes descritores: obesidade e covid-19; fatores de risco e Covid-19; obesidade e Sars-Cov-2 e suas versões em inglês.

Os critérios adotados de inclusão para as referências a serem utilizadas foram: artigos publicados nos idiomas português e inglês, disponíveis na íntegra, com acesso eletrônico livre e que correspondessem ao conteúdo da análise. Quanto aos de exclusão, foram descartados os estudos nos idiomas espanhóis, artigos repetidos, revisões bibliográficas e aqueles que não estivessem em consonância com a proposta desta produção ou que não se enquadraram nos critérios de inclusão mencionados a partir da leitura dos títulos e dos resumos de cada um dos artigos científicos selecionados.

\section{RESULTADOS E DISCUSSÃO}

A busca inicial resultou em 196 publicações as quais tiveram os seus respectivos resumos apreciados. Após filtragem, foram selecionados 45 estudos para uma leitura completa e selecionados 12 artigos como base para esta revisão bibliográfica. Desses, 6 estudos retrospectivos de coorte, 2 ensaios clínicos, um estudo retrospectivo de controle de caso, um estudo de coorte prospectivo, um estudo de diagnóstico e um estudo clínico multicêntrico retrospectivo.

Para sistematizar os achados, foi elaborada uma tabela contendo as principais características de cada artigo: autor e ano de publicação, tipo de estudo, população estudada e objetivo (Quadro 1). 
Quadro 1: Descrição dos estudos incluídos $(n=12)$.

\begin{tabular}{ll}
\hline Autor, ano. & Tipo de estudo \\
Cai SH. et al., 2020 & $\begin{array}{l}\text { Estudo clínico multicêntrico } \\
\text { retrospectivo. }\end{array}$
\end{tabular}

Chiappetta S. et al., Estudo de diagnóstico. 2020

Hamer M et al., 2020 Estudo de coorte.

Kalligeros M. et al., Estudo de coorte retrospectivo. 2020

Lemyze M. et al., Estudo retrospectivo de controle 2020 de caso.

Memtsoudis SG. et Ensaio clínico controlado al., 2020

Mostaghim A. et al., Estudo retrospectivo de coorte. 2020

Nakeshbandi M. et al., Estudo retrospectivo de coorte. 2020

\section{População estudada}

96 pacientes internados com infecção pelo SARS-CoV-2 foram matriculados no Hospital Do Povo de Dongguan, no hospital de Nanfang e no Primeiro Hospital Afiliado da Universidade de Xiamen.

33 pacientes com obesidade metabólica (EOSS 2 e 3 ).

Estudo populacional com dados de uma amostra de moradia comunitária na Inglaterra $(\mathrm{n}=334.329$; $56,4 \pm 8,1 \quad$ y; $54,5 \%$ mulheres) com potencial de ligação ao registro nacional de internação por COVID-19.

103 pacientes internados com COVID-19.
44 pacientes com síndrome de câncer respiratório agudo 2019 foram comparados com um grupo histórico de 39 pacientes consecutivos com síndrome de desconforto respiratório agudo admitidos na UTI pouco antes da crise da doença coronavírus 2019.

Dados de 113 pacientes com COVID-19 em três UTIs dos EUA.

791 pacientes com idade $\geq 18$ anos internados com teste positivo de PCR SARS-CoV-2. 684 pacientes com COVID-19.

\section{Objetivo}

Investigar se a obesidade desempenhou um papel nas manifestações clínicas e prognóstico em pacientes infectados com SARS-CoV-2.

Avaliar os fatores associados à anemia em gestantes atendidas pela rede pública de saúde de uma capital do Nordeste do Brasil.

Examinar associações entre IMC, obesidade central e COVID-19.

Explorar a potencial associação da obesidade e outras doenças crônicas com desfechos graves, como internação em Unidade de Terapia Intensiva (UTI) e Ventilação Mecânica Invasiva (IMV), em pacientes internados com COVID-19.

Investigar as características, o manejo e os desfechos da população gravemente doente admitido na UTI para a doença coronavírus de síndrome respiratória aguda grave 2019 causando uma síndrome de angústia respiratória aguda.

Avaliar a prevalência do uso de suplementação com ácido fólico em gestantes e identificar fatores associados à não realização dessa prática.

Caracterizar a associação entre desfechos clínicos em pacientes com obesidade e marcadores inflamatórios.

Elucidar a associação entre obesidade e desfechos COVID19. 
Peters SAE. et al., Estudo de coorte prospectivo. 2020

Simonnet A. et al., Estudo retrospectivo. 2020

Singh S. et al., $2020 \quad$ Estudo retrospectivo de coorte.

Steinberg E et al., Estudo de coorte retrospectivo. 2020

Análise de dados
retirados do UK do
Reino Unido.

Reino Unido.

124 pacientes consecutivos internados em tratamento intensivo para SARS-CoV-2 em um único centro francês.

Dados de prontuários eletrônicos de 41.513 pacientes adultos $(\geq 18$ anos), diagnosticados com COVID-19, de 26 organizações de saúde nos Estados Unidos. através da plataforma TriNetX.

210 pacientes com COVID-19.
Avaliar o risco de mortalidade por COVID-19 associada a várias medidas antropométricas em mulheres e homens.

Investigar a relação entre obesidade e síndrome respiratória aguda grave coronavírus-2 (SARS-CoV-2).

Avaliar os impactos da obesidade nos desfechos de pacientes internados com COVID-19.
Identificar se os desfechos adversos estão associados à obesidade, particularmente em pacientes COVID-19 com 45 anos ou mais.

Fonte: Autoria própria.

Cai e colaboradores (2020), ao investigarem se a obesidade poderia desenvolver algum papel nas manifestações clínicas e no prognóstico dos pacientes infectados com SARS-CoV-2, encontraram que o índice de massa corporal (IMC) estava significativamente relacionado com manifestações clínicas e resultados clínicos dos pacientes infectados por COVID-19.

Rocha e colaboradores corroboram enfatizando que existe associação entre obesidade e um pior prognóstico dos pacientes com infecção por SARS-Cov-2, deixando evidente que pacientes com sobrepeso e obesidade apresentam um maior risco de desenvolverem sintomas severos da doença, além de precisarem ser hospitalizados com maior frequência. Ademais, constatou-se que esses indivíduos tiveram uma maior admissão em unidades intensivas de cuidado (UTI) e na maioria dos casos, necessitaram de assistência respiratória assistida, além de verificar uma maior mortalidade por COVID-19 associada à obesidade.

Já Chiapeta e colaboradores (2020) alertam através de seus estudos que os médicos devem estar cientes de que a obesidade sendo classificada com base na estratificação de risco através do Sistema de Estadiamento da Obesidade de Edmonton (EOSS) que utiliza classificações médicas, mentais e/ou funcionais de pacientes, e não apenas com base no IMC, demonstrou melhor preditor da mortalidade por todas as causas e pode muito bem ser que os estágios da EOSS possam descrever melhor o risco de hiperinflamação em pacientes com infecção por COVID-19. Sendo que, a identificação precoce de uma possível grande inflamação possui papel fundamental na tomada de decisão em situações de hospitalização, 
suporte respiratório precoce e terapia com imunossupressão para melhora dos casos. Por esse mesmo lado, Hamer e colaboradores (2020) notaram uma maior probabilidade de COVID-19 e hospitalização em indíviduos com aumento da adiposidade geral e central, entrando em acordo com achados já citados.

Segundo Chait \& Hartigh (2020) a distribuição do tecido adiposo exibe grande importância nos indicadores de saúde, pois a gordura subcutânea vastamente expandida em indivíduos saudáveis, localizada na parte superior ou inferior da pele, é considerada com menor risco para o desenvolvimento de doenças crônicas, porém, quando essa reserva se acumula na parte visceral, contribui para a resistência à insulina, dislipidemia, aterosclerose, inflamação, diabetes II, síndrome metabólica e maior risco de doença cardiovascular (DCV).

Kalligeros e colaboradores (2020) observaram que a obesidade grave (IMC $\geq 35 \mathrm{~kg} / \mathrm{m} 2$ ) esteve associada à admissão na UTI da amostra pesquisada, e sugerem, ainda, a necessidade de maior vigilância e tratamento ativo de pacientes com obesidade e COVID-19. Nesse mesmo contexto, Mostaghim e colaboradores (2020), ao estudarem uma amostra com o objetivo de caracterizar a associação entre desfechos clínicos em pacientes com obesidade e marcadores inflamatórios, encontratam que, de 791 pacientes com Covid-19, 361 (45,6\%) tinham obesidade. Nas análises multivariadas realizadas, o alto grau de IMC manteve-se associado a uma maior chance de transferência para a UTI, além de mortalidade hospitalar, concluindo, assim, que pacientes com obesidade estariam mais propensos a ter maus resultados.

Lemyze e colaborades (2020) alegam que, em sua forma mais grave, a doença coronavírus 2019 atingiu, preferencialmente, a população obesa vulnerável, evoluindo para uma falência múltipla dos órgãos, ordenando apoio ventilatório mecânico delongado. Além disso, exigiu maior atenção e trabalho dos cuidadores. Concordando com Stavros e colaborades (2020) que, através de evidências preliminares, sugerem que a COVID-19 pode acarretar maiores prejuizos à saúde de idosos. Além disso, grande variedade de comorbidades é prevalente, a maioria notavelmente hipertensão, diabetes mellitus e doenças cardiovasculares, incluindo a obesidade.

Enfatizando sobre tais achados, Steinberg e colaboradores (2020), em um estudo de coorte retrospectivo, com 210 pacientes com COVID-19, notaram que todos os desfechos encontrados estiveram associados ao IMC elevado, concluindo que a obesidade esteve relacionada aos maus resultados em pacientes hospitalizados com essa doença.

Nakeshbandi e colaboradores (2020) apontam que o sobrepeso e a obesidade em pacientes acometidos com COVID-19 apresentaram risco aumentado de mortalidade e intubação quando comparados com aqueles que possuem IMC adequado.

Peters Sae e colaboradores (2020) observaram que o IMC esteve relacionado a um maior risco de mortalidade por COVID-19 em mulheres do que em homens, a razão de risco de mulheres para homens foi de 1,20 (intervalo de confiança de $95 \% 1,00 ; 1,43$ ). O estudo demonstrou o papel da obesidade na 
mortalidade de COVID-19 e mostra que os efeitos de um IMC elevado na mortalidade de COVID-19 podem ser mais fortes em mulheres do que homens. Por outro lado, os achados de Qingxian e colaboradores (2020) evidenciaram uma prevalência 42 vezes maior para o desenvolvimento de pneumonia grave comparando com aqueles em estado eutrófico, sendo o público masculino 4,4 vezes maior de progressão para a gravidade em relação ao feminino. De todo modo, chama-se a atenção para a obesidade manter-se associada a maiores complicações em diferentes estudos, em ambos os sexos.

Simonnet e colaboradores (2020) identificaram uma alta prevalência de pacientes obesos internados em terapia intensiva por SARS-CoV-2, sendo que, a gravidade da doença esteve aumentada de acordo com o IMC, concluindo que a obesidade é um fator de risco para o agravo da SARS-CoV-2, exigindo maior atenção às medidas preventivas nesses indivíduos.

Singh e colaboradores (2020) em seus estudos observaram que os pacientes com COVID-19, acometidos com qualquer grau de obesidade, tiveram um risco significativamente maior de internação e intubação ou morte em comparação com os pacientes sem obesidade. Os pacientes com obesidade severa apresentavam riscos ainda maiores de resultados ruins.

\section{CONSIDERAÇÕES FINAIS}

É percebido que a pandemia em decorrência dos quadros de obesidade é uma situação que pode ser controlada e reversível, uma vez que envolve, primordialmente, a adoção de mudanças no comportamento alimentar humano. No entanto, vale salientar, que por envolver questões socioculturais, e também emocionais e psicológicos, é preciso ter uma abordagem além de apenas controlar e orientar sobre questões alimentares exclusivamente.

Tendo visto que um dos fatores que mais pode influenciar, de maneira negativa, no prognóstico de pacientes portadores de obesidade, e que foram infectados pelo SARS-CoV-2, seja o quadro inflamado do paciente obeso, o presente estudo permite concluir que indíviduos portadores de obesidade possuem maiores chances de apresentarem complicações quando testados positivos para COVID-19, sendo que, estes, estão mais propensos a desenvolverem complicações durante o período de internação.

A literatura evidencia, de forma bem elucidada, o quanto é necessário a intensificação das orientações e acompanhamento de profissionais de saúde, com equipe multidisciplinar, no intuito de evitar a progressão e reduzir os resultados graves da COVID-19. Além disso, é preciso que sejam evidenciadas medidas pautadas no estilo de vida saudável para a melhora do estado nutricional.

Por fim, com base nos achados desse estudo, se faz pertinente a realização de estudos futuros a respeito do tema, a fim de possibilitar um melhor tratamento para pacientes enquadrados nesse cenário, e ainda, pela necessidade de examinar as características clínicas e os fatores de risco de pacientes com COVID-19 em populações grandes e diversas. 


\section{REFERÊNCIAS}

Abbas, A. M., Fathy, S. K., Fawzy, A. T., Salem, A. S., \& Shawky, M. S. (2020). Os efeitos mútuos de COVID-19 e obesidade. Remédio para obesidade, $19, \quad 100250$. https://doi.org/10.1016/j.obmed.2020.100250

Cai SH, Liao W, Chen SW, Liu LL, Liu SY, Zheng ZD. Associação entre obesidade e prognóstico clínico em pacientes infectados com SARS-CoV-2. Infectar a Pobreza. 2020 Jun 29;9(1):80. doi: 10.1186/s40249020-00703-5. PMID: 32600411; PMCID: PMC7322704.

Carneiro, A. B.; Dall'Igna, D. M. Obesidade como fator de risco para o agravamento da Covid-19: uma breve revisão de literatura. Vittalle - Revista de Ciências da Saúde v. 32, n. 3, p. 12-18, 2020.

Chait, A., den Hartigh, L. J. Adipose Tissue Distribution, Inflammation and Its Metabolic Consequences, Including Diabetes and Cardiovascular Disease. Front Cardiovasc Med. 7, 22. Published 2020 Feb 25. doi:10.3389/fcvm.2020.00022

Chiappetta, S., Sharma, A.M., Bottino, V. et al. COVID-19 e o papel da inflamação crônica em pacientes com obesidade. Int J Obes 44, 1790-1792 (2020). https://doi.org/10.1038/s41366-020-0597-4

Cummings, M. J., Baldwin, M. R., Abrams, D., Jacobson, S. D., Meyer, B. J., Balough, E. M., \& Hochman, B. R. (2020). Epidemiology, clinical course, and outcomes of critically ill adults with COVID-19 in New York City: a prospective cohort study. The Lancet. https://doi.org/10.1016/S0140-6736(20)31189-2

Dias PC, Henriques P, Anjos LA, Burlandy L. Obesidade e políticas públicas: concepções e estratégias adotadas pelo governo brasileiro. Cadernos de Saúde Pública 2017; 33(7): e00006016.

Hamer M, Gale CR, Kivimäki M, Batty GD. Overweight, obesity, and risk of hospitalization for COVID19: A community-based cohort study of adults in the United 331Kingdom. Proc Natl Acad Sci U S A. 2020.

Kalligeros M, Shehadeh F, Mylona EK, Benitez G, Beckwith CG, Chan PA, Mylonakis E. Association of Obesity with Disease Severity Among Patients with Coronavirus Disease 2019. Obesidade (Primavera da Prata). 2020 Jul;28(7):1200-1204. doi: 10.1002/oby.22859. Epub 2020 Jun 12. 32352637; PMCID: PMC7267224.

Lemyze M, Courageux N, Maladobry T, Arumadura C, Pauquet P, Orfi A, Komorowski M, Mallat J, Granier M. Implications of Obesity for the Management of Severe Coronavirus Disease 2019 Pneumonia. Crit Care Med. 2020 Set;48(9):e761-e767. doi: 10.1097/CCM.000000000000455. PMID: 32452889; PMCID: PMC7255399.

Melo, S. P. S. C.; Cesse, E. A. P.; Lira, P. I. C.; Ferreira, L. C. C. N.; Rissin, A.; Batista Filho, M. Sobrepeso, obesidade e fatores associados aos adultos em uma área urbana carente do Nordeste Brasileiro. Rev. Bras. Epidemiol., v. 23, p. 1-14, 2020. doi: 10.1590/1980-549720200036

Memtsoudis SG, Ivascu NS, Pryor KO, Goldstein PA. Obesidade como fator de risco para o mau resultado na lesão pulmonar induzida pelo COVID-19: o papel potencial da apneia obstrutiva obstrutiva do sono não diagnosticada. Br J Anaesth. 2020;125(2):e262-e263. doi:10.1016/j.bja.2020.04.078 
Mostaghim A, Sinha P, Bielick C, et al. Desfechos clínicos e níveis de marcadores inflamatórios em pacientes com Covid-19 e obesidade em um hospital de rede de segurança do centro da cidade. PLos Um. 2020;15(12):e0243888. Publicado em 2020 Dez 16. doi:10.1371/journal.pone.0243888

Nakeshbandi M, Maini R, Daniel P, Rosengarten S, Parmar P, Wilson C, Kim JM, Oommen A, Mecklenburg M, Salvani J, Joseph MA, Breitman I. O impacto da obesidade nas complicações do COVID19: estudo retrospectivo de coorte. Int J Obes (Lond). 2020 Set;44(9):1832-1837. doi: 10.1038/s41366-0200648-x. Epub 2020 Jul 25. PMID: 32712623; PMCID: PMC7382318.

Qingxian, C., Chen, F., Fang, L., Xiaohui, L., Tao, W., Qikai, W. Q., Zhaoqin, W., Liu, Y., a Jun, C., Lei, L., Lin, X., Obesity and COVID-19 Severity in a Designated Hospital in Shenzhen, China (3/13/2020). https://doi.org/.2139/ssrn.

Peters SAE, MacMahon S, Woodward M. Obesity as a risk factor for COVID-19 mortality in women and men in the UK biobank: Comparisons with influenza/pneumonia and coronary heart disease. Diabetes Obes Metab. 2021 Jan;23(1):258-262. doi: 10.1111/dom.14199. Epub 2020 Oct 11. PMID: 32969132; PMCID: PMC7536945.

Rocha GV, Soares CEM, Filho LOH, Amaral MVF, Castro VE, Júnior EA, Ribeiro KA, Amâncio NFG. A influência da obesidade na mortalidade de adultos com COVID-19. Brazilian Journal of Health Review, Curitiba, v.4, n.1, p.1405-1418 Jan/Feb. 2021

SBP-Sociedade Brasileira de Pediatria Departamento de Nutrologia Obesidade na infância e adolescência: Manual de Orientação. São Paulo: SBP; 2019.

Simonnet A, Chetboun M, Poissy J, Raverdy V, Noulette J, Duhamel A, Labreuche J, Mathieu D, Pattou F, Jourdain M; LICORN e o grupo de estudo Lille COVID-19 e Obesity. Alta prevalência de obesidade em Síndrome Respiratória Aguda Grave Coronavirus-2 (SARS-CoV-2) necessitando de ventilação mecânica invasiva. Obesidade (Primavera da Prata). 2020 Jul;28(7):1195-1199. doi: 10.1002/oby.22831. Epub 2020 Jun 10. Errata em: Obesidade (Primavera de Prata). 2020 Out;28(10):1994. PMID: 32271993; PMCID: PMC7262326.

Singh S, Bilal M, Pakhchanian H, Raiker R, Kochhar GS, Thompson CC. Impact of Obesity on Outcomes of Patients With Coronavirus Disease 2019 in the United States: A Multicenter Electronic Health Records Network Study. Gastroenterologia. 2020;159(6):2221-2225.e6. doi:10.1053/j.gastro.2020.08.028

Steinberg E, Wright E, Kushner B. Em Adultos Jovens com COVID-19, a obesidade está associada a desfechos adversos. West J Emerg Med. v. 21, n. 4, p. 752-755, 2020. doi:10.5811/westjem.2020.5.47972

World Health Organization. Body mass index classification - report of a WHO consultation on obesity. Geneva: WHO; 1995. 